\title{
Synthesis, characterization, sintering and dielectric properties of nanostructured perovskite-type oxide, $\mathrm{Ba}_{2} \mathbf{G d S b O}_{6}$
}

\author{
C VIJAYAKUMAR, H PADMA KUMAR ${ }^{\dagger}$, SAM SOLOMON ${ }^{\dagger \dagger}$, J K THOMAS ${ }^{\dagger}$, \\ P R S WARRIAR* and J KOSHY ${ }^{\dagger}$ \\ Department of Physics, University College, Trivandrum 695 034, India \\ ${ }^{\dagger}$ Electronic Materials Research Laboratory, Department of Physics, Mar Ivanios College, \\ Trivandrum 695 015, India \\ ${ }^{\dagger \dagger}$ Department of Physics, St. Johns College, Anchal 691 306, India
}

MS received 24 November 2007; revised 16 April 2008

\begin{abstract}
Nanoparticles of barium gadolinium antimonate $\left(\mathrm{Ba}_{2} \mathbf{G d S b O}_{6}\right)$, a complex perovskite-type oxide, has been synthesized using an auto ignition combustion process for the first time. The nanoparticles thus obtained have been characterized by powder $X$-ray diffraction, thermogravimetric analysis, differential thermal analysis, Fourier transform infrared spectroscopy and transmission electron microscopy. The XRD studies have shown that the as-prepared powder is phase pure $\mathrm{Ba}_{2} \mathbf{G d S b O}_{6}$ and has a complex cubic perovskite $\left(\mathrm{A}_{2} \mathrm{BB}^{\prime} \mathrm{O}_{6}\right)$ crystalline structure with lattice constant, $a=8.449 \AA$. The TEM image reveals that the particle size of the as-prepared nano powder was in the range $30-60 \mathrm{~nm}$. The nanocrystals of $\mathrm{Ba}_{2} \mathrm{GdSbO}_{6}$ synthesized by the combustion technique could be sintered to $96 \%$ of the theoretical density by heating at a temperature of $1560^{\circ} \mathrm{C}$ for a short duration of $3 \mathrm{~h}$. The surface morphology of the sintered pellet has been studied by scanning electron microscope (SEM). The dielectric constant $\left(\varepsilon_{\mathrm{r}}\right)$ was 20 and the loss factor $(\tan \delta)$ was 0.03 at $3 \mathrm{MHz}$. By the present combustion technique a phase pure nanopowder of $\mathrm{Ba}_{2} \mathrm{GdSbO}_{6}$ could be obtained by a single step process without the need of any calcination step.
\end{abstract}

Keywords. Samarium barium antimonite; nanoparticles; combustion synthesis; sintering.

\section{Introduction}

Materials with perovskite structure exhibit a wide range of properties due to their diversity of crystal structures (Kimura et al 2000; Litchenberg et al 2001). $\mathrm{Ba}_{2} \mathrm{LnSbO}_{6}$ ( $\mathrm{Ln}=$ lanthanides) compounds with double perovskite $\mathrm{A}_{2} \mathrm{BB}^{\prime} \mathrm{O}_{6}$ structure have been extensively studied in the past (Blasse 1965; Garcia Cascado et al 1984; Alonso et al 1997; Karunadasa et al 2003). Among these materials, $\mathrm{Ba}_{2} \mathrm{GdSbO}_{6}$ compound was of special interest because of its applications (Kurian et al 1995) as substrate for high temperature superconducting $\mathrm{YBa}_{2} \mathrm{Cu}_{3} \mathrm{O}_{7-\delta}$ films. The crystal structure of $\mathrm{Ba}_{2} \mathrm{GdSbO}_{6}$ material was reported as double cubic perovskite (Kurian et al 1995). In all the above studies, conventional solid state synthesis route was used for the preparation of their single phase compounds which involved prolonged calcination at high temperatures $\left(>1200^{\circ} \mathrm{C}\right.$ for $\left.24 \mathrm{~h}\right)$ with intermediate grindings, yielding large coarse grained micron sized powders. The coarse-grained powders synthesized using the conventional solid state route have the disadvantages of larger particle size, high temperature processing and relatively

*Author for correspondence (prswariar@yahoo.com) lower phase purity (Hoffler and Averback 1990; McCandlish et al 1992).

The synthesis of advanced ceramic materials as nanoparticles is currently gaining widespread interest in materials research (Karagedov and Lyakhov 1999; Ravichandran et al 1999; Gleiter 2000). The advantages of nanocrystalline materials are superior phase homogeneity, sinterability and microstructure leading to unique mechanical, electrical, dielectric, magnetic, optical and catalytic properties. The combustion synthesis is a processing technique currently being used as a route for the production of engineering ceramics and other advanced materials in their nano powder form and this process provides energy and cost saving advantages over the other processing routes (Saha and Pramanik 1997; Patil et al 2001; James et al 2004). In this paper, synthesis, characterization, sintering and dielectric properties of phase pure $\mathrm{Ba}_{2} \mathrm{GdSbO}_{6}$ nanoparticles using a single step modified combustion process are reported for the first time.

\section{Experimental}

The basic requirement for the synthesis of an oxide material by the chemical route is that it should be possible to 
obtain all the ions involved in solution before initiating further reactions. Therefore, for the preparation of $\mathrm{Ba}_{2} \mathrm{GdSbO}_{6}$, it is necessary to have an aqueous solution of $\mathrm{Ba}, \mathrm{Gd}$ and $\mathrm{Sb}$ ions. To get a clear solution containing the $\mathrm{Gd}, \mathrm{Ba}$ and $\mathrm{Sb}$ ions, $\mathrm{Gd}_{2} \mathrm{O}_{3}$ was dissolved in dilute nitric acid, $\mathrm{Ba}\left(\mathrm{NO}_{3}\right)_{2}$ in water, $\mathrm{Sb}_{2} \mathrm{O}_{3}$ in boiling tartaric acid and they were mixed together. Aqueous solution containing ions of $\mathrm{Gd}, \mathrm{Ba}, \mathrm{Sb}$ were prepared from high purity $\mathrm{Gd}_{2} \mathrm{O}_{3}\left(99.9 \%\right.$, $\mathrm{CDH}$, India), $\mathrm{Ba}\left(\mathrm{NO}_{3}\right)_{2}(99.9 \%, \mathrm{CDH}$, India) and $\mathrm{Sb}_{2} \mathrm{O}_{3}$ (purified, Merck). To get the precursor complex, citric acid was added to the solution containing the metal ions, maintaining the citric acid to the cation ratio at unity. The oxidant-fuel ratio of the system was adjusted by using nitric acid and ammonium hydroxide, and the ratio was kept at unity. The solution containing the complex precursor mixture at a $\mathrm{pH}$ of $\sim 7$ was heated using a hot plate at $\sim 250^{\circ} \mathrm{C}$ in a ventilated fume hood. The solution boils on heating and undergoes dehydration and decomposition leading to a smooth deflation and producing foam. The foam then ignites by itself on persistent heating giving voluminous and fluffy product of combustion. The combustion product was subsequently characterized as single-phase nanocrystals of $\mathrm{Ba}_{2} \mathrm{GdSbO}_{6}$. Structure of the as-prepared powder was examined by powder X-ray diffraction (XRD) technique using a X-ray Diffractometer (Model Bruker D-8) with Nickel filtered $\mathrm{CuK}_{\alpha}$ radiation. The differential thermal analysis (DTA) and thermogravimetric analyses (TGA) were carried out using Perkin-Elmer TG/DT thermal analyser in the range $30-1100^{\circ} \mathrm{C}$ at a heating rate of $20^{\circ} \mathrm{C} / \mathrm{min}$ in nitrogen atmosphere. The infrared (IR) spectra of the samples were recorded in the range $400-4000 \mathrm{~cm}^{-1}$ on a Thermo-Nicolet Avatar 370 Fourier transform infrared (FTIR) spectrometer using $\mathrm{KBr}$ pellet method. Particulate properties of the combustion product were examined using transmission electron microscopy (TEM, Model-Hitachi H-600, Japan) operating at $200 \mathrm{kV}$. The samples for transmission electron microscope (TEM) were prepared by ultrasonically dispersing the powder in methanol and allowing a drop of this to dry on a carbon-coated copper grid. The selected area diffraction (SAD) pattern is also recorded for a camera length of $1.35 \mathrm{~m}$. To study the sinterability of the nanoparticles obtained by the present combustion method, the asprepared $\mathrm{Ba}_{2} \mathrm{GdSbO}_{6}$ nanoparticles were mixed with $5 \%$ polyvinyl alcohol and pressed in the form of cylindrical pellet of $14 \mathrm{~mm}$ diameter and $\sim 2 \mathrm{~mm}$ thickness at a pressure of about $350 \mathrm{MPa}$ using a hydraulic press. The pellet was then sintered at $1560^{\circ} \mathrm{C}$ for $3 \mathrm{~h}$. The theoretical density of the $\mathrm{Ba}_{2} \mathrm{GdSbO}_{6}$ was calculated from the lattice constants and sintered density was determined using Archimedes method. The surface morphology and energy dispersive X-ray analysis of the sintered samples were examined using scanning electron microscopy (SEM, Model-Hitachi S 2400 Japan). For low frequency dielectric studies the pellets were made in the form of a disc capacitor with the specimen as the dielectric medium. Both flat surfaces of the sintered pellet were polished and then electroded by applying silver paste. The capacitance of the samples were measured using an LCR meter (Hioki$3532-50$ ) for the frequency range $100 \mathrm{~Hz}-3 \mathrm{MHz}$.

\section{Results and discussion}

Figure 1 shows the XRD pattern of the as-prepared powder obtained by the present combustion synthesis. The broad peak in figure 1(a) at $d=2.98726 \AA$ corresponds to the major (220) reflections of $\mathrm{Ba}_{2} \mathrm{GdSbO}_{6}$. All the peaks are indexed for a complex cubic $(\mathrm{Fm} 3 \mathrm{~m})$ perovskite structure and the lattice constant of $\mathrm{Ba}_{2} \mathrm{GdSbO}_{6}$ determined from XRD pattern is $a=8.449 \AA$, which agrees very well with the reported values (Kurian et al 1995). The crystallite size calculated from the full width at half maximum (FWHM) using Scherrer formula for the major (220) peak in figure 1(a) is $\sim 23 \mathrm{~nm}$. The as-prepared powder was heated in the range $600^{\circ} \mathrm{C}$ for $1 \mathrm{~h}$. The XRD patterns of the sample heated at $600^{\circ} \mathrm{C}$ is shown in figure $1(\mathrm{~b})$. No structural changes were observed in the heated samples as compared to as-prepared powder, which indicates that the formation of $\mathrm{Ba}_{2} \mathrm{GdSbO}_{6}$ phase was complete during the combustion process itself without a need for any calcination step. It may be noted that single phase $\mathrm{Ba}_{2} \mathrm{GdSbO}_{6}$ powder can be obtained via the solid state reaction route, only after calcining the reaction mixture at $1200^{\circ} \mathrm{C}$ for $24 \mathrm{~h}$ with three intermediate grindings (Kurian et al 1995). The present method is the successful one for the preparation of single phase $\mathrm{Ba}_{2} \mathrm{GdSbO}_{6}$ without the need for any further calcination or high temperature annealing procedure. In other combustion processes reported for the preparation of nano crystals of some ceramic oxides, polyvinyl alcohol and urea were used as chelating agents and fuel, respectively and in all these cases phase pure powder was obtained only after high temperature annealing (Saha and

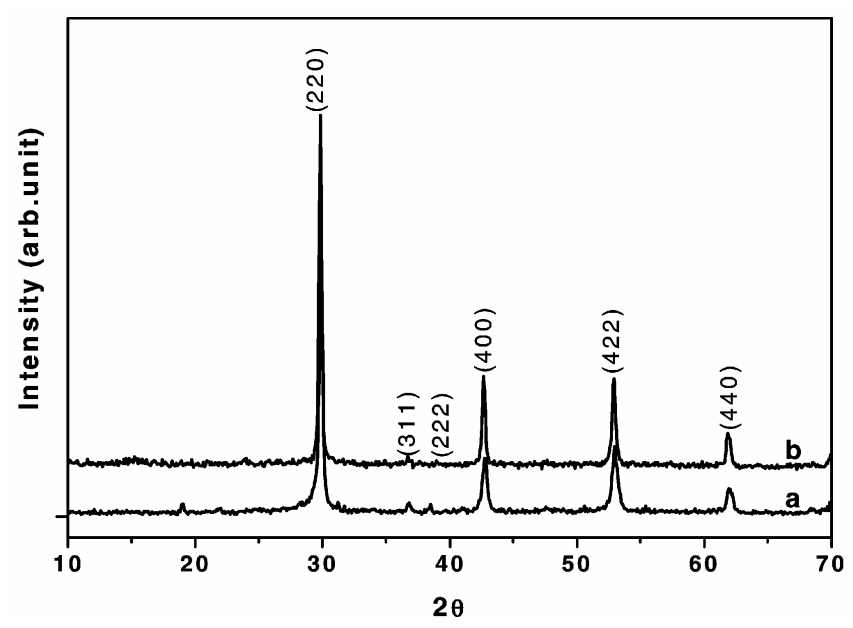

Figure 1. XRD pattern of a. as-prepared $\mathrm{Ba}_{2} \mathrm{GdSbO}_{6}$ nanocrystals and $\mathbf{b}$. heated at $600^{\circ} \mathrm{C}$. 
Pramanik 1997; Patil et al 2001). But in the present modified combustion method, citric acid was used as the chelating agent instead of polyvinyl alcohol and urea was replaced with ammonia. By changing the complexing agent and oxidant fuel system it was possible to get a single phase $\mathrm{Ba}_{2} \mathrm{GdSbO}_{6}$ as nanocrystals in a single step combustion.

A typical DTA and TGA curves of the as-prepared $\mathrm{Ba}_{2} \mathrm{GdSbO}_{6}$ powder up to $1100^{\circ} \mathrm{C}$ are shown in figure 2 . The TGA curve shows a weight loss of $<3 \%$ which can be caused by the liberation of adsorbed moisture in the sample and other than this, there is no weight or enthalpy change occurring in the sample at high temperatures up to $1100^{\circ} \mathrm{C}$, which implies that the combustion is complete and no organic matter is present in the sample. There is no evidence of any phase transition taking place in the sample up to a temperature of $1100^{\circ} \mathrm{C}$.

Figure 3 shows FT-IR spectrum of the typical as-prepared $\mathrm{Ba}_{2} \mathrm{GdSbO}_{6}$ nanopowder prepared through combustion synthesis. All the peaks in figure 3 are characteristics of

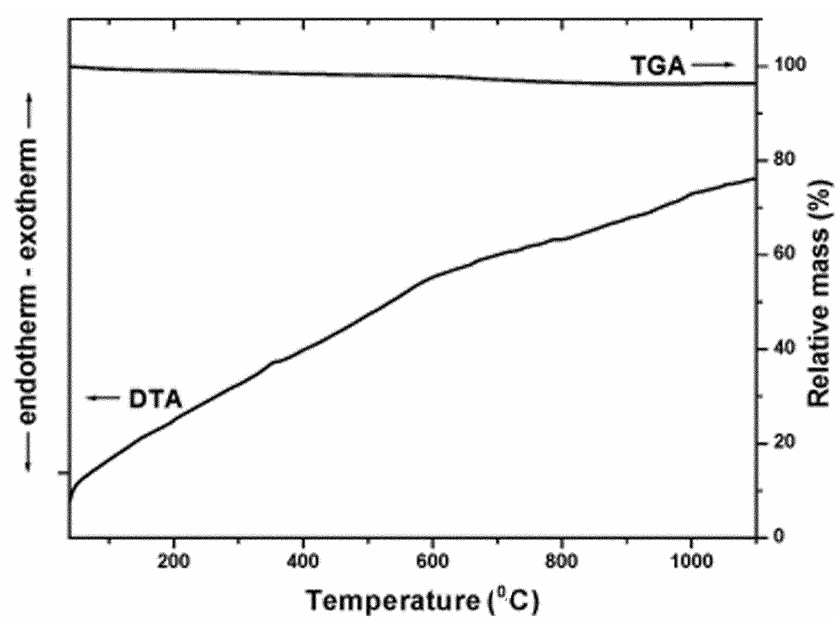

Figure 2. DTA/TGA trace of as-prepared $\mathrm{Ba}_{2} \mathrm{GdSbO}_{6}$ nanocrystals.

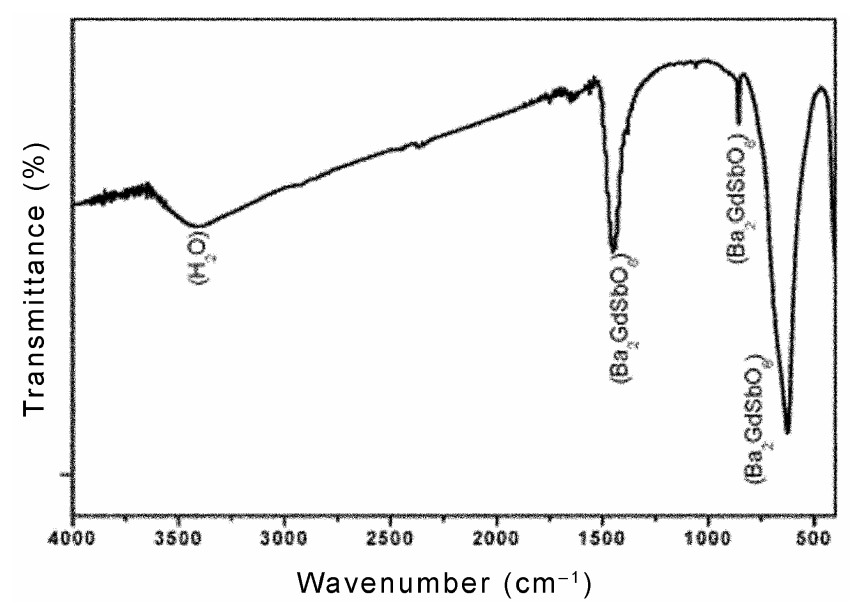

Figure 3. FT-IR spectrum of $\mathrm{Ba}_{2} \mathrm{GdSbO}_{6}$ powder obtained through combustion synthesis. the material except the one at $3415 \mathrm{~cm}^{-1}$ which is due to the presence of adsorbed moisture. This corroborates the $\mathrm{XRD}$ and thermal analysis result that the combustion is complete and no organic matter is present in the sample.

The transmission electron microscopic (TEM) studies on the powder morphology of the as-prepared $\mathrm{Ba}_{2} \mathrm{GdSbO}_{6}$ nanopowder obtained by the combustion synthesis showed that the nanoparticles are of submicron size 30-60 nm with a mean size of $40 \mathrm{~nm}$ as shown in figure 4 with regular

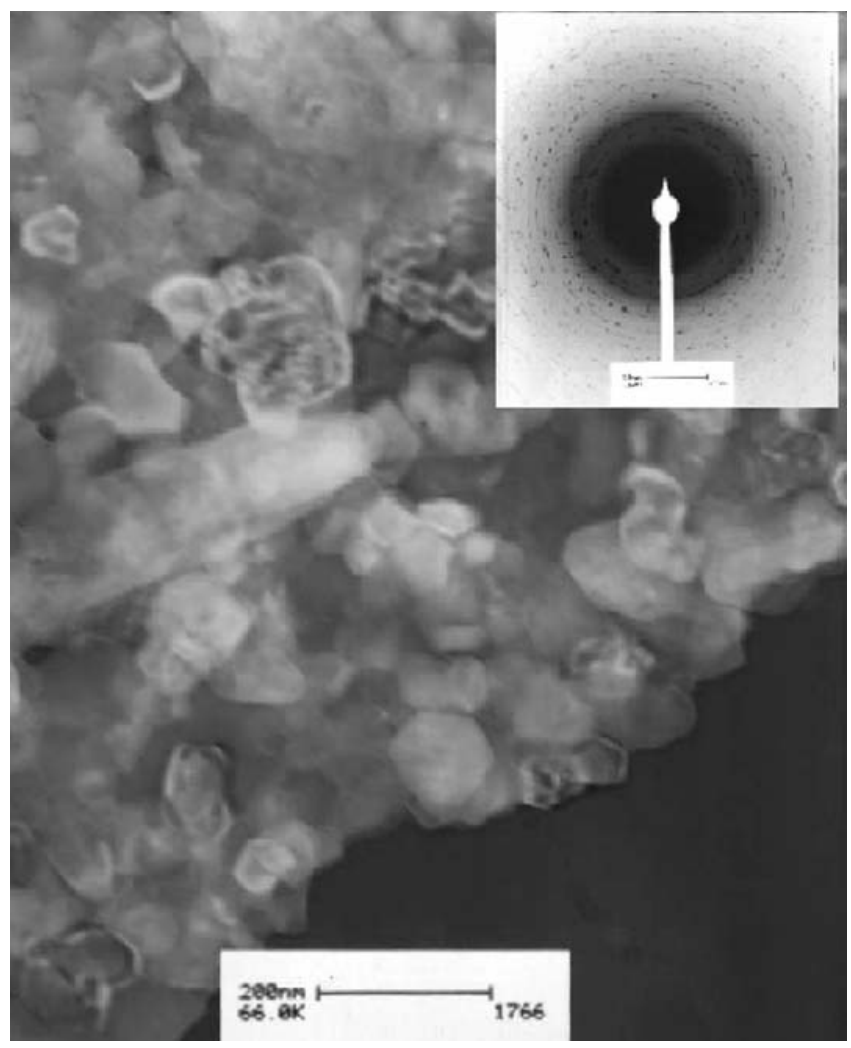

Figure 4. TEM micrographs of as prepared $\mathrm{Ba}_{2} \mathrm{GdSbO}_{6}$ nano powder in bright field and corresponding electron diffraction pattern.

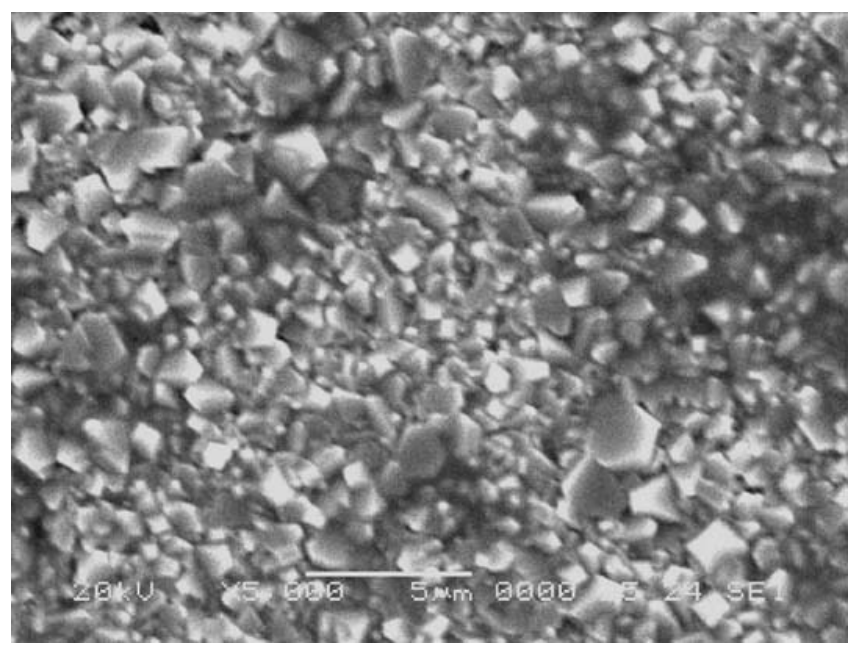

Figure 5. SEM micrograph of the $\mathrm{Ba}_{2} \mathrm{GdSbO}_{6}$ sintered pellet. 
hexagonal shape. Figure 4 is a typical TEM bright field image of the as prepared powder and the inset is the corresponding selected area electron diffraction pattern (SAD). The particles are of regular shape with sharp grain boundaries. The ring nature of the electron diffraction pattern is indicative of the polycrystalline nature of the crystallites, but the spotty nature of the SAD pattern can be due to the fact that the finer crystallites having related orientations are agglomerated together resulting in a limited set of orientations.

The sintering behaviour of the nanocrystals of $\mathrm{Ba}_{2} \mathrm{GdSbO}_{6}$ powders synthesized through the present combustion route was studied. The relative green density of the specimen used for the sintering study was $55 \%$ and sintered density was $>96 \%$ of the theoretical value. Compared to the material prepared through the conventional route, the sintering duration was found to be reduced considerably from $12 \mathrm{~h}$ to $3 \mathrm{~h}$. Surface morphology of the sintered $\mathrm{Ba}_{2} \mathrm{GdSbO}_{6}$ specimen was examined using SEM and is shown in figure 5 . It is clear from the micrograph that densification was achieved without significant microstructural coarsening. Average grain size determined from the SEM micrograph is $\sim 500 \mathrm{~nm}$.

Figure 6 shows the variation of dielectric constant $\left(\varepsilon_{\mathrm{r}}\right)$ and loss factor $(\tan \delta)$ of the sintered pellet measured in the frequency range $100 \mathrm{~Hz}-3 \mathrm{MHz}$. The study revealed that the dielectric constant $\left(\varepsilon_{\mathrm{r}}\right)$ does not show significant variation with frequency and it falls from 26-20 in the measured frequency range of $100 \mathrm{~Hz}-3 \mathrm{MHz}$. The loss factor $(\tan \delta)$ also gets decreased when the frequency is increased from $100 \mathrm{~Hz}-3 \mathrm{MHz}$ and has a value of 0.03 at a frequency of $3 \mathrm{MHz}$ at room temperature. The low values of dielectric constant $\left(\varepsilon_{\mathrm{r}}\right)$ and loss factor $(\tan \delta)$ fulfils one of the most important requirements of a material to be used as a substrate for the fabrication of high $T_{\mathrm{c}}$ superconducting films.

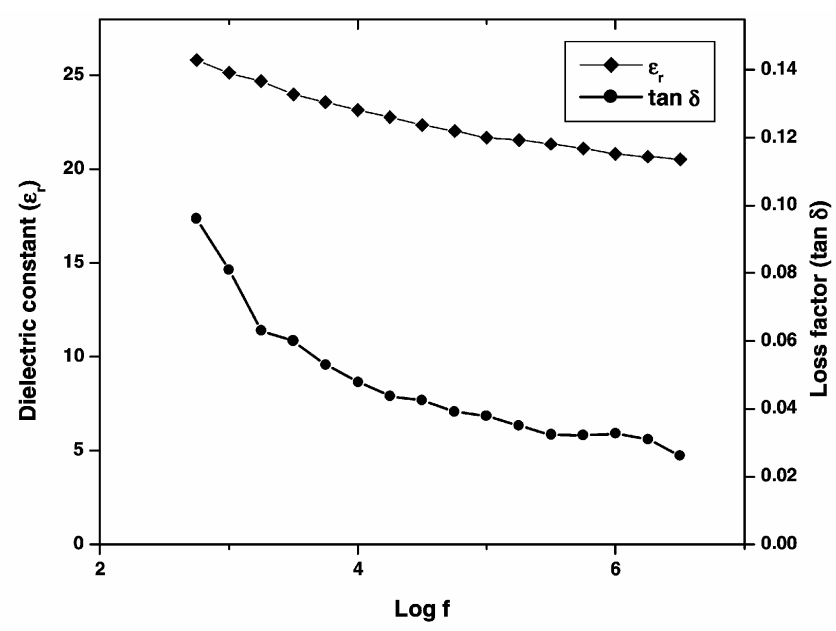

Figure 6. Variation of dielectric constant $\left(\varepsilon_{\mathrm{r}}\right)$ and loss factor $(\tan \delta)$ with frequency.

\section{Conclusions}

$\mathrm{Ba}_{2} \mathrm{GdSbO}_{6}$ has been synthesized as single phase nano crystals $(30-60 \mathrm{~nm})$ via an exothermic chemical process for the first time. The solid combustion product obtained was characterized by X-ray diffraction (XRD), electron diffraction, differential thermal analysis (DTA), thermogravimetric analysis (TGA), infrared spectroscopy (FT-IR) and transmission electron microscope (TEM). X-ray diffraction and electron diffraction have shown that the asprepared powder is phase pure $\mathrm{Ba}_{2} \mathrm{GdSbO}_{6}$ and has a complex cubic perovskite $\mathrm{A}_{2} \mathrm{BB}^{\prime} \mathrm{O}_{6}$ structure with lattice constant, $a=8.449 \AA$. DTA and TGA studies have shown that there is no phase transition taking place in the asprepared powder.

The transmission electron microscopy image of the asprepared powder reveals that the particle size is in the range $30-60 \mathrm{~nm}$. The nanopowder could be sintered to a density of $96 \%$ of the theoretical density at $1560^{\circ} \mathrm{C}$ for $3 \mathrm{~h}$. The SEM image of the sintered material indicates high densification of the material with average grain size of $500 \mathrm{~nm}$. The room temperature dielectric constant $\left(\varepsilon_{\mathrm{r}}\right)$ and loss factor $(\tan \delta)$ of the sintered pellet at $3 \mathrm{MHz}$ was 20 and 0.03 , respectively which indicates that the material is suitable for microwave applications. The combustion synthesis has an advantage that the phase pure $\mathrm{Ba}_{2} \mathrm{GdSbO}_{6}$ could be obtained by a single step process without the need of any calcination step.

\section{References}

Alonso J A, Cascales C, Garcia Cascado P and Rasines I 1997 J. Solid State Chem. 128247

Blasse G 1965 J. Inorg. Nucl. Chem. 27993

Garcia Cascado P, Mendola A and Rasines L 1984 Z. Anorg. Allg. Chem. $\mathbf{5 1 0} 194$

Gleiter H 2000 Acta Mater. 481 and references therein

Hoffler H J and Averback R S 1990 Scr. Metall. Mater. 24240

James J, Jose R, John A M and Koshy J 2004 US Patent No. 6, 761,866

Karagedov G R and Lyakhov N Z 1999 Nanostruct. Mater. 11 559

Karunadasa H, Huang Q, Ueland B G, Schiffer P and Cava R J 2003 PNAS 1008097

Kimura T and Tokura Y 2000 Ann. Rev. Mater. Sci. 30451

Kurian J, Koshy J, Wariar P R S, Yadava Y P and Damodaran A D 1995 J. Solid State Chem. 1161993

Litchenberg F, Herrnberger A, Weidenman K and Mannhart J 2001 Prog. Solid State Chem. 291

McCandlish L E, Kear B H and Kim B K 1992 Nanostruct. Mater. 1119

Patil R C, Radhakrishnan S, Sushama Pethkar and Vijayamohanan K 2001 J. Mater. Res. 167

Ravichandran D, Roy R, Ravindranathan P and White W B 1999 J. Am. Ceram. Soc. 821082

Saha S K and Pramanik P 1997 Nanostruct. Mater. 829 